\title{
Lopsided diffractions of distinct symmetries in two-dimensional non-Hermitian optical gratings
}

\author{
Yi-Mou Liu, ${ }^{1}$ Feng Gao, ${ }^{2}$ Jin-Hui Wu,,${ }^{1,}{ }^{*}$ M. Artoni, ${ }^{3}$ and G. C. La Rocca ${ }^{4}$ \\ ${ }^{1}$ School of Physics, Northeast Normal University, Changchun 130024, China \\ ${ }^{2}$ College of Physics, Jilin University, Changchun 130012, China \\ ${ }^{3}$ Department of Engineering and Information Technology and Istituto Nazionale di Ottica (INO-CNR), \\ Brescia University, 25133 Brescia, Italy \\ ${ }^{4}$ Scuola Normale Superiore and CNISM, 56126 Pisa, Italy
}

(Received 19 April 2019; published 2 October 2019)

\begin{abstract}
Fraunhofer light diffraction across a thin two-dimensional lattice of cold atoms subject to transverse hybrid modulations of two standing-wave crossed pump fields is seen to yield lopsided patterns of various degrees of symmetry. We show that one can fully restrain the diffraction of a weak incident probe to two diagonal or adjacent quadrants or even just to a single quadrant, depending on the phases of two standing-wave pumps and on the probe detuning. Different diffraction symmetries with respect to the axes or diagonals of the diffraction plane quadrants are interpreted here in terms of different out-of-phase interplay of absorption and dispersion periodic distributions, resulting from different combinations of Hermitian, $\mathcal{P} \mathcal{T}$-symmetric, and non-Hermitian modulations.
\end{abstract}

DOI: 10.1103/PhysRevA.100.043801

\section{INTRODUCTION}

Non-Hermitian optical systems with parity-time $(\mathcal{P} \mathcal{T})$ symmetry [1-4] and antisymmetry [5-9] have attracted a great deal of attention because they provide possibilities for controlling photon flows with various interesting characteristics. Optical $\mathcal{P} \mathcal{T}$ symmetry typically requires the complex refractive index to satisfy the condition $n(x)=n^{*}(-x)$ in a given direction. As opposed to $\mathcal{P} \mathcal{T}$ symmetry, $\mathcal{P} \mathcal{T}$ antisymmetry is realized in optical media with the complex refractive index satisfying instead the condition $n(x)=-n^{*}(-x)$. Recent work has reported that $\mathcal{P} \mathcal{T}$ symmetry and antisymmetry can simultaneously occur in the same optical structure [10-12], whereas feasible ways to convert from one to the other are discussed in [8,9]. More importantly, non-Hermitian optical structures have been explored to extend fascinating studies of optical Bloch oscillation [13,14], coherent perfect absorption [15], photon or phonon lasing [16-19], etc., and have become the basis of a few nonreciprocal optical phenomena such as unidirectional reflection [20,21], unidirectional invisibility [22,23], and asymmetric diffraction [24-26]. In particular, complex crystals described by non-Hermitian Hamiltonians with complex periodic potentials have been expected to host spectral singularities whose signatures could be assessed through a typical Bragg diffraction experiment [27].

Applying standing-wave (SW), instead of traveling-wave (TW), control fields in atomic media subject to electromagnetically induced transparency (EIT) [28] is an efficient way to realize complex periodic potentials that lay at the basis of well-known phenomena such as dynamically tunable photonic band gaps (PBGs) [29-31] and stationary light pulses (SLPs) [32-34]. This method has also been used to realize structures

\footnotetext{
*jhwu@nenu.edu.cn
}

of electromagnetically induced gratings (EIGs) [35-38] with special forms of spatially periodic absorption and dispersion. One main advantage of such Hermitian EIG structures is that intensity distributions of the diffracted photons among different orders can be manipulated "on demand" through flexible amplitude and/or phase modulations of the transmission function. This may be explored to generalize earlier works on the lopsided diffraction of atomic (optical) waves off one-dimensional (1D) non-Hermitian optical (atomic) gratings with fixed potentials $[39,40]$. In fact, unconventional optical modulations on EIG structures have recently been brought to the development of cooperative nonlinear gratings and non-Hermitian gratings. Cooperative nonlinear gratings allow one to distinguish light fields of different photon statistics with the dipole blockade effect of Rydberg atoms [41,42], while non-Hermitian gratings typically result in asymmetric diffraction patterns that can be tuned through the out-of-phase interplay of phase and amplitude modulations [43-46]. Unidirectional and controlled higher-order diffraction, through nonHermitian modulations on EIG structures built from Rydberg atoms driven beyond the dipole blockade regime, has also been reported [47]. Most of the above works hinge, however, on 1D Hermitian or non-Hermitian EIG structures.

Here we investigate instead a two-dimensional (2D) nonHermitian EIG structure consisting of a square optical lattice filled with ultracold atoms driven into the four-level $\mathcal{N}$ configuration (see Fig. 1) by two TW pumps and two orthogonal SW pumps (2D pump cross gratings) [see Fig. 2(a)]. This realizes a thin 2D non-Hermitian grating that, under specific driving conditions, enables one to attain an arbitrary combination of Hermitian, $\mathcal{P} \mathcal{T}$-symmetry, and non-Hermitian modulations on the probe absorption and dispersion along the two orthogonal lattice axes. Such modulations, which may be "pure" or "hybrid", result in peculiar diffraction patterns bearing double diagonal, single axial, and single diagonal symmetries in the 


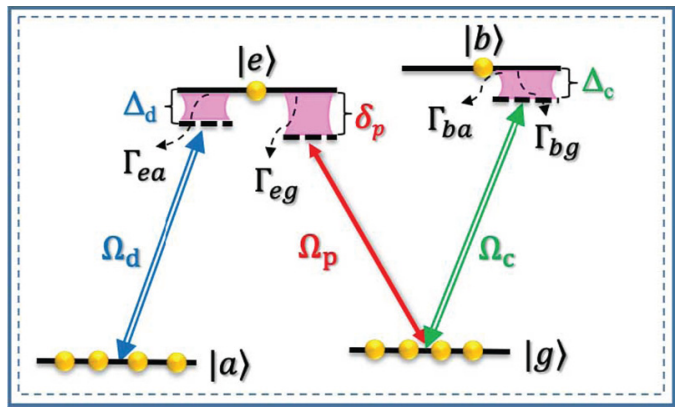

FIG. 1. A four-level $\mathcal{N}$ configuration for ${ }^{87} \mathrm{Rb}$ atoms driven by a probe field of Rabi frequency (detuning) $\Omega_{p}\left(\delta_{p}\right)$ and two pump fields of Rabi frequencies (detunings) $\Omega_{d}$ and $\Omega_{c}\left(\Delta_{d}\right.$ and $\Delta_{c}$ ). The four levels are $|g\rangle \equiv\left|5 S_{1 / 2}, F=1\right\rangle,|a\rangle \equiv\left|5 S_{1 / 2}, F=2\right\rangle,|e\rangle \equiv$ $\left|5 P_{1 / 2}, F=1\right\rangle$, and $|b\rangle \equiv\left|5 P_{1 / 2}, F=2\right\rangle$ with decay rates $\Gamma_{e g}=$ $\Gamma_{b g}=\Gamma_{e a}=\Gamma_{b a} \simeq 2 \pi \times 5.9 \mathrm{MHz}$.

diffraction plane. Consequently, we observe diffracted photons only in two diagonal quadrants, two adjacent quadrants, or a single quadrant, while diffraction patterns can be made to undergo a $\pi / 2$-rotation sweep by just changing the probe detuning and/or the pump phases. It is finally worth mentioning that the lopsided diffraction mechanism we propose here may turn out to be relevant to the development of new concepts on wave-front shaping that are now attained instead through metasurfaces with subwavelength resolutions [48-50].

This work is organized through the following Sec. II, where we summarize the background model, and Sec. III, where we discuss the far-field Fraunhofer diffraction resulting from three types of pure or hybrid modulations and interpret the results as arising from the out-of-phase interplay between real (dispersion) and imaginary (absorption) parts of the probe susceptibility. We summarize, at last, our conclusions in Sec. IV.

\section{THE MODEL}

We start by considering the four-level $\mathcal{N}$ configuration of cold ${ }^{87} \mathrm{Rb}$ atoms driven by three coherent fields of frequencies (amplitudes) $\omega_{p}\left(\mathcal{E}_{p}\right), \omega_{c}\left(\mathcal{E}_{c}\right)$, and $\omega_{d}\left(\mathcal{E}_{d}\right)$, as shown in Fig. 1. The weak probe field $\omega_{p}$ interacts with transition $|g\rangle \leftrightarrow|e\rangle$, while the strong pump fields $\omega_{c}$ and $\omega_{d}$ act upon transitions $|g\rangle \leftrightarrow|b\rangle$ and $|a\rangle \leftrightarrow|e\rangle$, respectively. The corresponding detunings (Rabi frequencies) are defined as $\delta_{p}=\omega_{p}-\omega_{e g}$

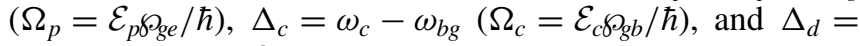
$\omega_{d}-\omega_{e a}\left(\Omega_{d}=\mathcal{E}_{d \wp_{a e}} / \hbar\right)$, with $\omega_{i j}$ being transition frequencies and $\wp_{i j}$ dipole moments. The atoms are assumed to be loaded into a square optical lattice of period $a$ along both the $x$ and $y$ axes [see Fig. 2(a)]. Around the intensity maxima formed by two (Gaussian) counterpropagating red-detuned laser beams, a depth minimum occurs, leading to an approximately harmonic lattice potential trap [51]. Assuming that (i) all lattice traps are equally populated and (ii) the trapped atoms are cool enough to occupy the lowest energy levels, we represent the atomic density distribution at each (trap) site $\left\{x_{i}, y_{i}\right\}$ as

$$
N_{i, j}(x, y)=N_{0} e^{-\left[\left(x-x_{i}\right)^{2} / \sigma_{x}^{2}+\left(y-y_{j}\right)^{2} / \sigma_{y}^{2}\right]},
$$

corresponding to the ground state of a $2 \mathrm{D}$ (harmonic) trap [52]. Here, $N_{0}$ is the (average) peak density, while $\sigma_{x}$ and $\sigma_{y}$ are the (average) half widths of a Gaussian distribution along the transverse trapping directions [53]. The above assumptions enable us to introduce the periodic susceptibility exhibited by the incident probe,

$$
\chi_{p}(x, y)=\frac{\gamma_{g e}^{2}}{2 \varepsilon_{0} \hbar \Omega_{p}} \sigma_{g e} \sum_{i, j} N_{i, j}(x, y)=\alpha \sigma_{g e} N(x, y),
$$

with $\alpha=\wp \rho_{g e}^{2} / 2 \varepsilon_{0} \hbar \Omega_{p}$. The polarization $\sigma_{g e}$ can be obtained by solving the density matrix equations

$$
\begin{aligned}
\partial_{t} \sigma_{g g}= & \Gamma_{b g} \sigma_{b b}+\Gamma_{e g} \sigma_{e e}+i \Omega_{c}^{*} \sigma_{b g}-i \Omega_{c} \sigma_{g b} \\
& +i \Omega_{p}^{*} \sigma_{e g}-i \Omega_{p} \sigma_{g e} \\
\partial_{t} \sigma_{a a}= & \Gamma_{b a} \sigma_{b b}+\Gamma_{e a} \sigma_{e e}+i \Omega_{d}^{*} \sigma_{e a}-i \Omega_{d} \sigma_{a e}, \\
\partial_{t} \sigma_{b b}= & -\Gamma_{b a} \sigma_{b b}-\Gamma_{b g} \sigma_{b b}+i \Omega_{c} \sigma_{g b}-i \Omega_{c}^{*} \sigma_{b g}, \\
\partial_{t} \sigma_{g a}= & -\gamma_{g a}^{\prime} \sigma_{g a}+i \Omega_{c}^{*} \sigma_{b a}+i \Omega_{p}^{*} \sigma_{e a}-i \Omega_{d} \sigma_{g e}, \\
\partial_{t} \sigma_{g b}= & -\gamma_{g b}^{\prime} \sigma_{g b}+i \Omega_{c}^{*} \sigma_{e b}+i \Omega_{c}^{*}\left(\sigma_{b b}-\sigma_{g g}\right), \\
\partial_{t} \sigma_{g e}= & -\gamma_{g e}^{\prime} \sigma_{g e}+i \Omega_{c}^{*} \sigma_{b e}+i \Omega_{p}^{*}\left(\sigma_{e e}-\sigma_{g g}\right)-i \Omega_{d}^{*} \sigma_{g a}, \\
\partial_{t} \sigma_{a b}= & -\gamma_{a b}^{\prime} \sigma_{a b}+i \Omega_{d}^{*} \sigma_{e b}-i \Omega_{c}^{*} \sigma_{a g},
\end{aligned}
$$

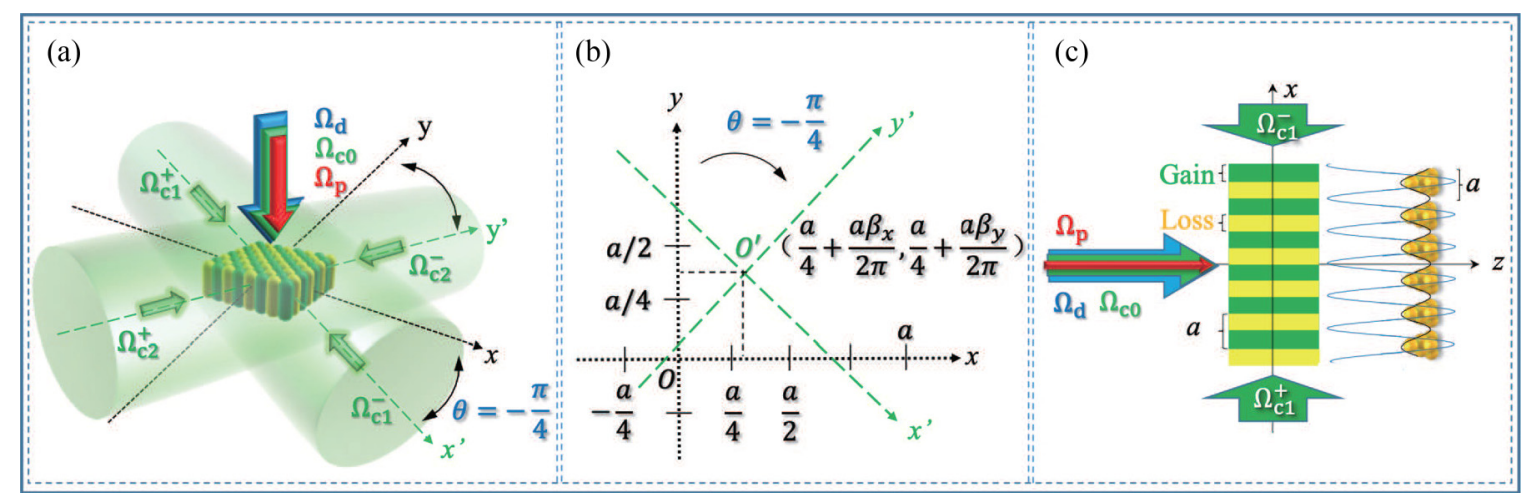

FIG. 2. (a) Non-Hermitian 2D pump grating along the $x^{\prime}$ and $y^{\prime}$ axes (period $a / \sqrt{2}$ ) modulating a 2D atomic lattice along the $x$ and $y$ axes (period $a$ ) with $\Omega_{c 0}, \Omega_{c 1}^{ \pm}$, and $\Omega_{c 2}^{ \pm}$together taking the role of $\Omega_{c}$ in Fig. 1. (b) Details of the $O(x, y)$ and $O^{\prime}\left(x^{\prime}, y^{\prime}\right)$ coordinate systems of relative orientations given in terms of their origins and axes. (c) A side view of the $2 \mathrm{D}$ grating, i.e., its projection in the $x z$ plane with balanced gain (green) and loss (yellow) in the $\mathcal{P} \mathcal{T}$-symmetry regime. 


$$
\begin{aligned}
& \partial_{t} \sigma_{a e}=-\gamma_{a e}^{\prime} \sigma_{a e}-i \Omega_{p}^{*} \sigma_{a g}+i \Omega_{d}^{*}\left(\sigma_{e e}-\sigma_{a a}\right), \\
& \partial_{t} \sigma_{b e}=-\gamma_{b e}^{\prime} \sigma_{b e}+i \Omega_{c} \sigma_{g e}-i \Omega_{p}^{*} \sigma_{b g}-i \Omega_{d}^{*} \sigma_{b a},
\end{aligned}
$$

which arise as usual from the interaction Hamiltonian, in the rotating-wave and electric-dipole approximations, associated with our $\mathcal{N}$-type driving configuration,

$$
\begin{aligned}
\mathcal{H}_{I}= & \hbar\left[\delta_{p} \hat{\sigma}_{e e}+\left(\delta_{p}-\Delta_{d}\right) \hat{\sigma}_{a a}+\Delta_{c} \hat{\sigma}_{b b}\right. \\
& +\hbar\left[\Omega_{p} \hat{\sigma}_{e g}+\Omega_{d} \hat{\sigma}_{e a}+\Omega_{c} \hat{\sigma}_{b g}+\text { H.c. }\right]
\end{aligned}
$$

Here, $\hat{\sigma}_{\mu \nu}=|\mu\rangle\langle\nu|$ define the projection $(\mu=v)$ and transition $(\mu \neq v)$ operators [54], and their expectation values $\sigma_{\mu \mu}$ and $\sigma_{\mu \nu}$ denote the atomic population at level $|\mu\rangle$ and atomic coherence between levels $|\mu\rangle$ and $|\nu\rangle$, respectively. They satisfy the properties $\sum_{\mu} \sigma_{\mu \mu}=1$ and $\sigma_{\mu \nu}=\sigma_{\nu \mu}^{*}$, while $\gamma_{\mu \nu}=\sum_{k}\left(\Gamma_{\mu k}+\Gamma_{\nu k}\right) / 2$ denote the dephasing rates, with
$\Gamma_{e g}$ and $\Gamma_{e a}\left(\Gamma_{b g}\right.$ and $\left.\Gamma_{b a}\right)$ being the decay rates from level $|e\rangle(|b\rangle)$ to levels $|g\rangle$ and $|a\rangle$, respectively. We also introduce the multiphoton detunings $\Delta_{g a}=\delta_{p}-\delta_{d}, \Delta_{b e}=\delta_{p}-$ $\delta_{c}$, and $\Delta_{a b}=\delta_{c}+\delta_{d}-\delta_{p}$, as well as the complex dephasing rates $\gamma_{g a}^{\prime}=\gamma_{g a}+i \Delta_{g a}, \gamma_{g b}^{\prime}=\gamma_{g b}+i \delta_{c}, \gamma_{g e}^{\prime}=\gamma_{g e}+i \delta_{p}$, $\gamma_{a b}^{\prime}=\gamma_{a b}+i \Delta_{a b}, \gamma_{a e}^{\prime}=\gamma_{a e}+i \delta_{d}$, and $\gamma_{b e}^{\prime}=\gamma_{b e}+i \Delta_{b e}$, for convenience in Eq. (3).

The real $\chi_{p}^{R}(x, y)$ and imaginary $\chi_{p}^{I}(x, y)$ parts in Eq. (2) describe the probe dispersion and absorption properties, respectively. When $\Omega_{c, d}$ and $\delta_{c, d}$ are "constant", changes of $\chi_{p}^{R}(x, y)$ and $\chi_{p}^{I}(x, y)$ along both the $x$ and $y$ axes occur "in phase". This would lead to traditional 2D EIG structures exhibiting symmetric diffraction patterns [56,57]. However, nontrivial 2D EIG structures may be attained if changes of $\chi_{p}^{R}(x, y)$ and $\chi_{p}^{I}(x, y)$ along the $x$ and/or $y$ axes can be set to occur "not in phase", e.g., through the following crossedpump modulation:

$$
\begin{aligned}
\Omega_{c}(x, y) & =\Omega_{c 0}+\Omega_{c 1}\left(x^{\prime}\right)+\Omega_{c 2}\left(y^{\prime}\right)=\Omega_{c 0}+\frac{1}{2} \delta \Omega_{c} \cos \frac{x^{\prime}}{a / 2 \sqrt{2} \pi}+\frac{1}{2} \delta \Omega_{c} \cos \frac{y^{\prime}}{a / 2 \sqrt{2} \pi} \\
& =\Omega_{c 0}+\frac{1}{2} \delta \Omega_{c} \cos \frac{(x-\delta x)-(y-\delta y)}{a / 2 \pi}+\frac{1}{2} \delta \Omega_{c} \cos \frac{(x-\delta x)+(y-\delta y)}{a / 2 \pi} \\
& =\Omega_{c 0}+\delta \Omega_{c} \cos \frac{(x-\delta x)}{a / 2 \pi} \cos \frac{(y-\delta y)}{a / 2 \pi}=\Omega_{c 0}+\delta \Omega_{c} f(x, y) .
\end{aligned}
$$

Here, $(\delta x, \delta y)$ denote the amounts by which the maxima of Rabi frequency $\Omega_{c}(x, y)$ shift relative to the corresponding maxima of density distribution $N(x, y)$. We denote by $\Omega_{c 0}$ a constant TW component, while $\Omega_{c 1}\left(x^{\prime}\right)$ and $\Omega_{c 2}\left(y^{\prime}\right)$ are two SW components of period $a / \sqrt{2}$ along the two orthogonal axes $x^{\prime}$ and $y^{\prime}$ rotated by an angle $\theta=-\pi / 4$ relative to the $x y$ plane [see Figs. 2(a) and 2(b)]. For convenience, in the following discussion, we further rewrite the modulating factor as

$$
f(x, y)=\sin \left(\frac{x}{a / 2 \pi}-\beta_{x}\right) \sin \left(\frac{y}{a / 2 \pi}-\beta_{y}\right)
$$

by rescaling $\delta x=a / 4+a \beta_{x} / 2 \pi$ and $\delta y=a / 4+a \beta_{y} / 2 \pi$. The off-center cross modulation in Eqs. (5) and (6) thus results in a space-dependent probe coherence $\sigma_{g e}(x, y)$ which, together with $N(x, y)$ in Eq. (2), yields nontrivial modulations on our atomic lattice, whose diffraction features will be discussed in the next section. A side view of the 2D lattice under the $\mathcal{P} \mathcal{T}$-symmetry modulation has been shown in Fig. 2(c) to gain a clearer impression.

For a weak probe impinging perpendicular to the thin atomic lattice [55], shown in Fig. 2(a), the (far-field) 2D diffraction intensity [56,57] can be written as

$$
\begin{aligned}
I_{p}\left(\theta_{x}, \theta_{y}\right) \approx & \left|E_{p}^{I}\left(\theta_{x}, \theta_{y}\right)\right|^{2} \\
& \times\left[\frac{\sin \left(\pi M R \sin \theta_{x}\right)}{M \sin \left(\pi R \sin \theta_{x}\right)} \frac{\sin \left(\pi M R \sin \theta_{y}\right)}{M \sin \left(\pi R \sin \theta_{y}\right)}\right]^{2},
\end{aligned}
$$

where the geometric factors depend on two ratios $R=a / \lambda_{p}$ and $M=w_{o} / a$, with $w_{o}$ being the width of the incident probe beam. We have also denoted by $\theta_{x}$ and $\theta_{y}$ the diffraction angles with respect to the $z$ direction in the $x z$ and $y z$ planes.
More specifically, probe diffraction will take place in a few directions determined by the diffraction order $\{m, n\}$ according to angles $\theta_{x} \rightarrow \theta_{x, m}=\arcsin (m / R)$ and $\theta_{y} \rightarrow \theta_{y, n}=$ $\arcsin (n / R)$, with the number of diffraction orders depending on the ratio $R$. The single square lattice (i.e., 2D unit cell) diffraction function,

$$
E_{p}^{I}\left(\theta_{x}, \theta_{y}\right)=\int_{-\frac{a}{2}}^{\frac{a}{2}} \int_{-\frac{a}{2}}^{\frac{a}{2}} T_{L}(x, y) e^{-i 2 \pi R\left(x \sin \theta_{x}+y \sin \theta_{y}\right)} d x d y,
$$

depends instead on the probe dispersion and absorption directly through the transmission

$$
T_{L}(x, y)=e^{-2 \pi L \chi_{p}^{I}(x, y) / \lambda_{p}} e^{i 2 \pi L \chi_{p}^{R}(x, y) / \lambda_{p}}
$$

across a short distance $L$ in the $z$ direction [58,59]. Note that phase and amplitude modulations in Eq. (9) are associated, respectively, with $\chi_{p}^{R}(x, y)$ and $\chi_{p}^{I}(x, y)$, whose control makes it feasible to attain the modulations on $\chi_{p}^{R}(x, y)$ and $\chi_{p}^{I}(x, y)$ that are "not in phase" that we sought for. We will discuss the corresponding nontrivial modulations on our atomic lattice in the next section.

\section{RESULTS AND DISCUSSION}

Now we examine and discuss the far-field Fraunhofer diffraction that results from driving our $2 \mathrm{D}$ atomic lattice both with (A) a $\mathcal{P} \mathcal{T}$-symmetry modulation and (B) a hybrid non-Hermitian modulation. We just consider the partial $\mathcal{P} \mathcal{T}$ symmetry with respect to the $x \rightarrow-x$ and $y \rightarrow y$ transformation or the $x \rightarrow x$ and $y \rightarrow-y$ transformation by plotting the diffraction intensity $I_{p}\left(\theta_{x}, \theta_{y}\right)$ for nearly vanishing pump detunings. 

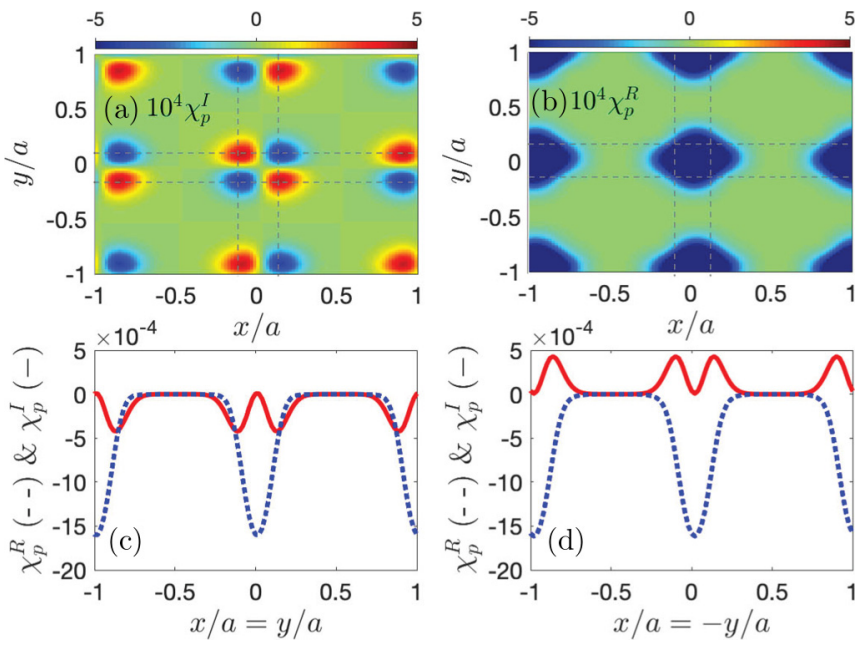

FIG. 3. (a) Absorption and (b) dispersion distributions of a 2D $\mathcal{P} \mathcal{T}$-symmetry atomic lattice attained for $\beta_{x}=\beta_{y}=0$, together with their 1D projections along the (c) $x=y$ and (d) $x=-y$ directions. Other parameters are $\Omega_{d}=2.0 \mathrm{MHz}, \Omega_{c 0}=4.5 \mathrm{MHz}, \delta \Omega_{c}=$ $1.6 \mathrm{MHz}, \delta_{p}=5.96 \mathrm{MHz}, R=4, M=10, L=100 \mu \mathrm{m}$, and $N_{0}=$ $3.5 \times 10^{11} \mathrm{~cm}^{-3}$.

\section{A. $\mathcal{P} \mathcal{T}$-symmetry modulation}

Our 2D pump cross grating works in the $\mathcal{P} \mathcal{T}$-symmetry regime when $f(x, y)$ is a product of sine functions with $\beta_{x}=$ $\beta_{y}=0$. It is clear that $\chi_{p}^{I}(x, y)$ and $\chi_{p}^{R}(x, y)$ are spatially out of phase (i.e., differ by $\pi / 2$ in phase at each point), with the former (latter) being an odd (even) function along the four gray lines in the $x$ and $y$ directions [see Figs. 3(a) and 3(b)]. In addition, $\chi_{p}^{I}(x, y)$ exhibits the double diagonal symmetry with two positive (negative) peaks in the $I I$ and $I V$ ( $I$ and III) quadrants in each period of the atomic lattice, while $\chi_{p}^{R}(x, y)$ exhibits the centrosymmetry with a single negative peak centered at the origin. This is further seen by extracting numerical values from the density plots of Figs. 3(a) and 3(b), namely, along the $x=y$ [see Fig. 3(c)] and $x=-y$ [see Fig. 3(d)] lines, respectively. The balanced gain for $x=y$ and loss for $x=-y$ (accompanied by the same dispersion) in the $2 \mathrm{D} \mathcal{P} \mathcal{T}$-symmetry regime can be thought of as the extension of the balanced gain regime for $x>0$ and loss for $x<-0$ (accompanied by the same dispersion) in the 1D $\mathcal{P} \mathcal{T}$-symmetry regime.

Figure 4 reports instead the diffraction intensity distribution for a $2 \mathrm{D} \mathcal{P} \mathcal{T}$-symmetry pump grating. We observe (i) diffracted photons only in the $I I$ and $I V$ quadrants, corresponding to regions where $\chi_{p}^{I}(x, y)>0$ (gain) and $\chi_{p}^{R}(x, y)<$ 0 (abnormal dispersion), with (ii) the diffraction pattern largely dependent on $N_{0}$, having more photons scattered into higher diffraction orders as $N_{0}$ increases. Therefore, $2 \mathrm{D} \mathcal{P} \mathcal{T}$ symmetry modulations lead to diffraction patterns of double diagonal symmetry with respect to the $x=y$ and $x=-y$ lines, with $N_{0}$ a crucial parameter to control how probe photons are distributed among different diffraction orders.

Finally, we address the question as to whether one may dynamically control "on demand" such an intriguing diffraction pattern. Figure 5 shows that the double diagonal symmetric diffraction, either in the $I I$ and $I V$ or in the $I$ and $I I I$
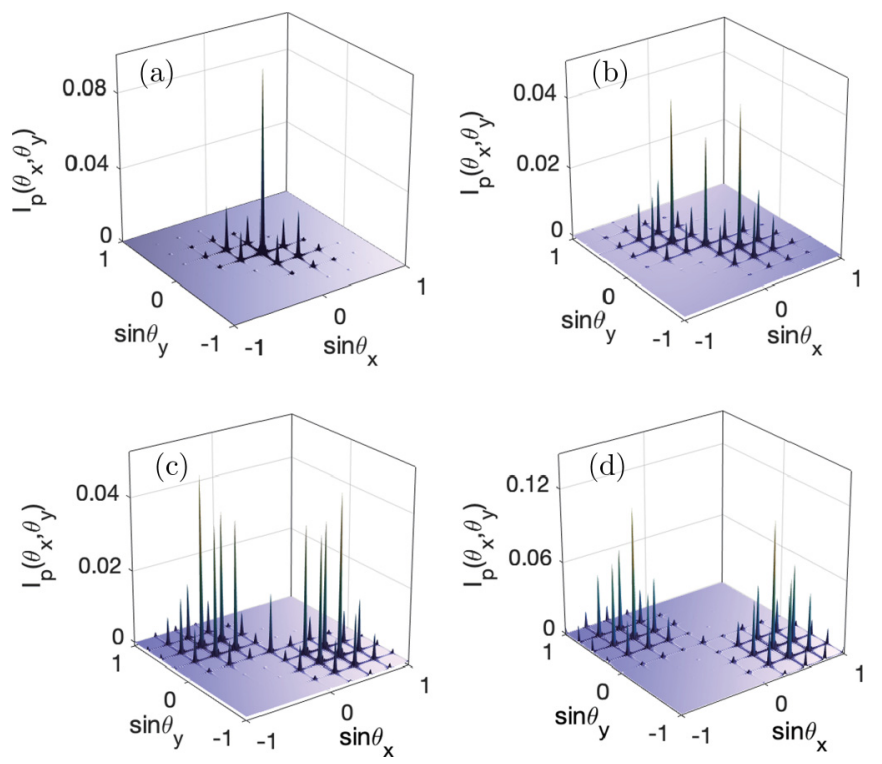

FIG. 4. Diffraction intensity $I_{p}\left(\theta_{x}, \theta_{y}\right)$ vs diffraction angles $\theta_{x}$ and $\theta_{y}$ for a $2 \mathrm{D} \mathcal{P} \mathcal{T}$-symmetry pump grating. Parameters are as in Fig. 3, except (a) $N_{0}=6.5 \times 10^{11} \mathrm{~cm}^{-3}$, (b) $8.7 \times 10^{11} \mathrm{~cm}^{-3}$, (c) $10.8 \times 10^{11} \mathrm{~cm}^{-3}$, and (d) $13.0 \times 10^{11} \mathrm{~cm}^{-3}$.

quadrants, depends on the signs of the probe detuning $\delta_{p}$ and the modulating factor $f(x, y)$, with the latter being controlled through the shifts $\beta_{x}$ and $\beta_{y}$ [60]. In particular, if we change the signs of both $\delta_{p}$ and $f(x, y)$ from " + " to " - ", the pattern remains bound to the $I I$ and $I V$ quadrants, yet when only one sign is changed, the whole pattern undergoes a $\pi / 2$ rotation
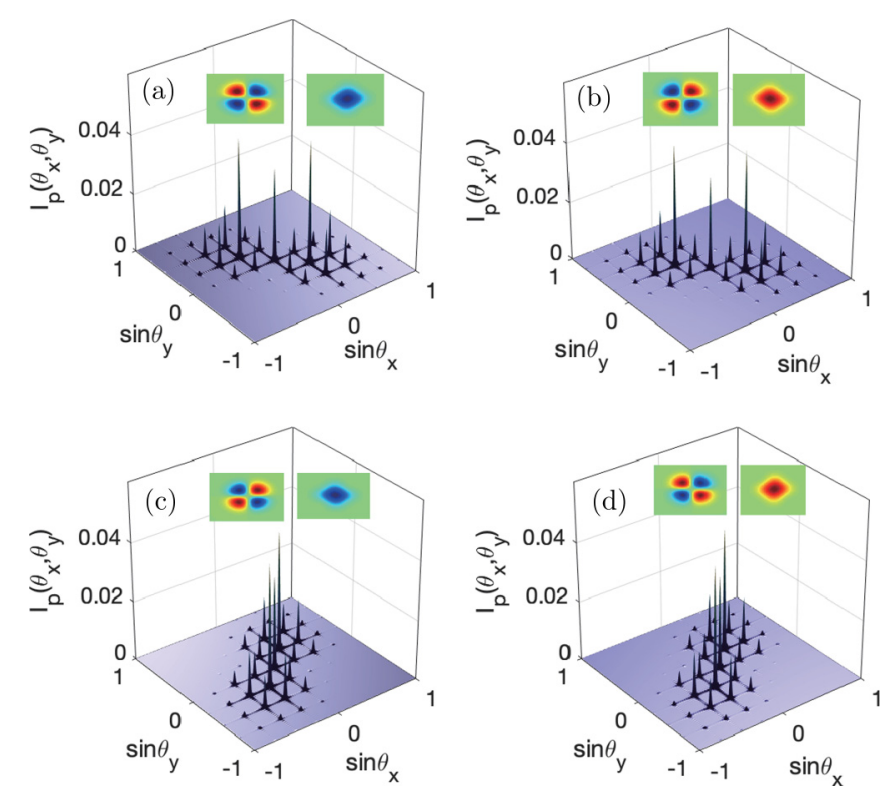

FIG. 5. Diffraction patterns of double diagonal symmetry attained for $\mathcal{P} \mathcal{T}$-symmetry modulations along both the $x$ and $y$ directions. Parameters are as in Fig. 3(b), except (a) $\delta_{p}=5.96 \mathrm{MHz}$ and $\beta_{y}=0$, (b) $\delta_{p}=-5.96 \mathrm{MHz}$ and $\beta_{y}=\pi$, (c) $\delta_{p}=5.96 \mathrm{MHz}$ and $\beta_{y}=\pi$, and (d) $\delta_{p}=-5.96 \mathrm{MHz}$ and $\beta_{y}=0$. The insets show the corresponding absorption (left) and dispersion (right) distributions in a single square lattice. 


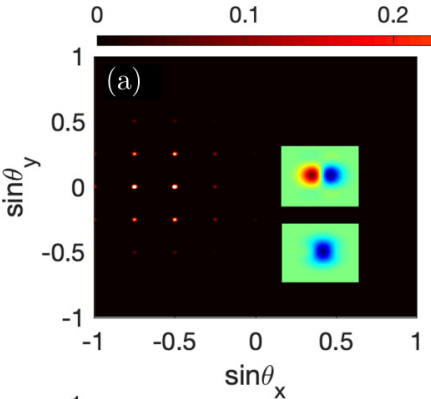

2

0.3
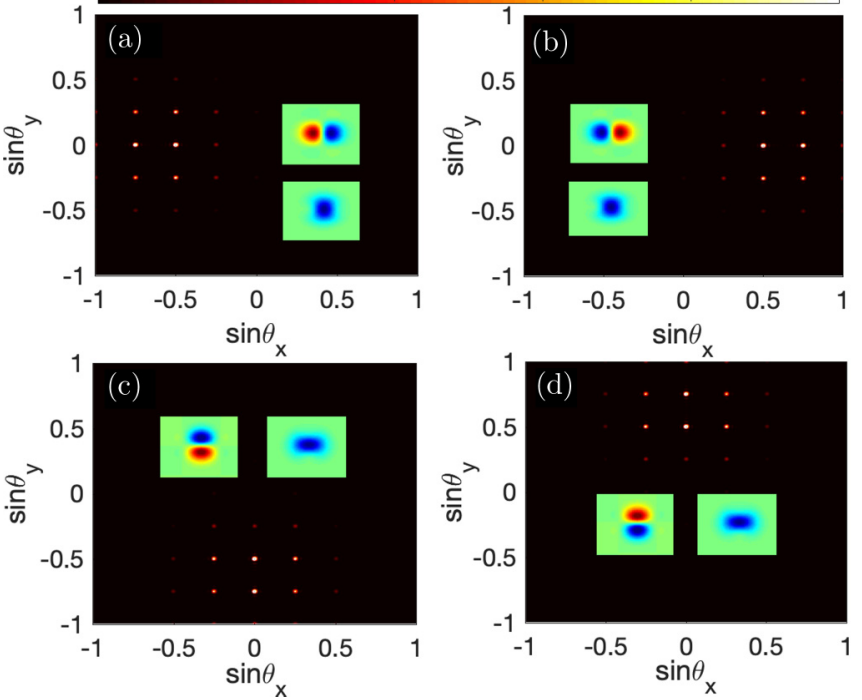

FIG. 6. Diffraction patterns of single axial symmetry attained for a $\mathcal{P} \mathcal{T}$-symmetry modulation along the $x$ (or $y$ ) direction and a Hermitian modulation along the $y$ (or $x$ ) direction. Parameters are as in Fig. 3(b), except that here (a) $\beta_{x}=0$ and $\beta_{y}=\pi / 2$, (b) $\beta_{x}=0$ and $\beta_{y}=-\pi / 2$, (c) $\beta_{y}=0$ and $\beta_{x}=\pi / 2$, and (d) $\beta_{y}=0$ and $\beta_{x}=$ $-\pi / 2$. The insets show the corresponding absorption (upper or left) and dispersion (lower or right) distributions in a single square lattice.

into the $I$ and $I I I$ quadrants. Because (i) the simultaneous sign change of $\delta_{p}$ and $f(x, y)$ results in the simultaneous sign change of $\chi_{p}^{I}(x, y)$ and $\chi_{p}^{R}(x, y)$, and (ii) the sign change of only $\delta_{p}$ or $f(x, y)$ results in the sign change of only $\chi_{p}^{I}(x, y)$ or $\chi_{p}^{R}(x, y)$, it is clear that the spatial interplay between the amplitude $\propto \chi_{p}^{I}(x, y)$ and phase $\propto \chi_{p}^{R}(x, y)$ modulations of the transmission $T_{L}(x, y)$ are responsible for this double diagonal symmetric type of diffraction. We may further conclude from the insets that probe photons are always diffracted into the quadrants where (1) $\chi_{p}^{I}(x, y)$ is positive (gain) when $\chi_{p}^{R}(x, y)$ is negative (anomalous dispersion) or where (2) $\chi_{p}^{I}(x, y)$ is negative (loss) when $\chi_{p}^{R}(x, y)$ is positive (normal dispersion).

\section{B. Hybrid non-Hermitian modulation}

We now consider two cases when our atomic lattice is driven by the $2 \mathrm{D}$ pump cross grating into a hybrid nonHermitian regime, whereby (i) a $\mathcal{P} \mathcal{T}$-symmetry (Hermitian) modulation is applied along the $x(y)$ or $y(x)$ axis, or (ii) different non-Hermitian modulations are applied along the $x$ and $y$ axes. A Hermitian modulation along the $x$ axis is attained by setting $\beta_{x}= \pm \pi / 2$, while a non-Hermitian modulation along the $x$ axis is attained by setting $\beta_{x}= \pm \pi / 4$ or $\pm 3 \pi / 4$ in the modulating factor $f(x, y)$; similarly when a Hermitian or non-Hermitian modulation along the $y$ axis is sought for.

Figure 6 shows that in case (i), probe photons are diffracted into adjacent quadrants, leading to single axial symmetric farfield diffraction. In particular, we have observed symmetric (asymmetric) diffraction in quadrants $I I$ and $I I I$ with respect to the $x(y)$ axis for $\beta_{x}=0$ and $\beta_{y}=\pi / 2$ [see Fig. 6(a)], quadrants $I$ and $I V$ with respect to the $x(y)$ axis for $\beta_{x}=0$ and $\beta_{y}=-\pi / 2$ [see Fig. 6(b)], quadrants $I I I$ and $I V$ with respect

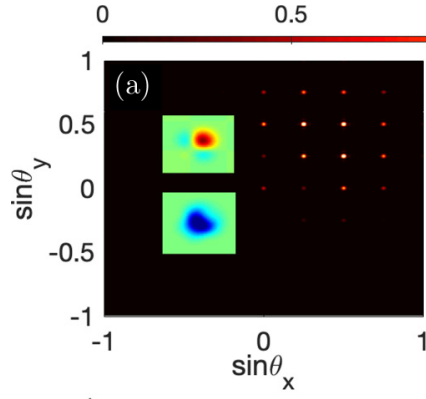

$1 \quad 1.5$
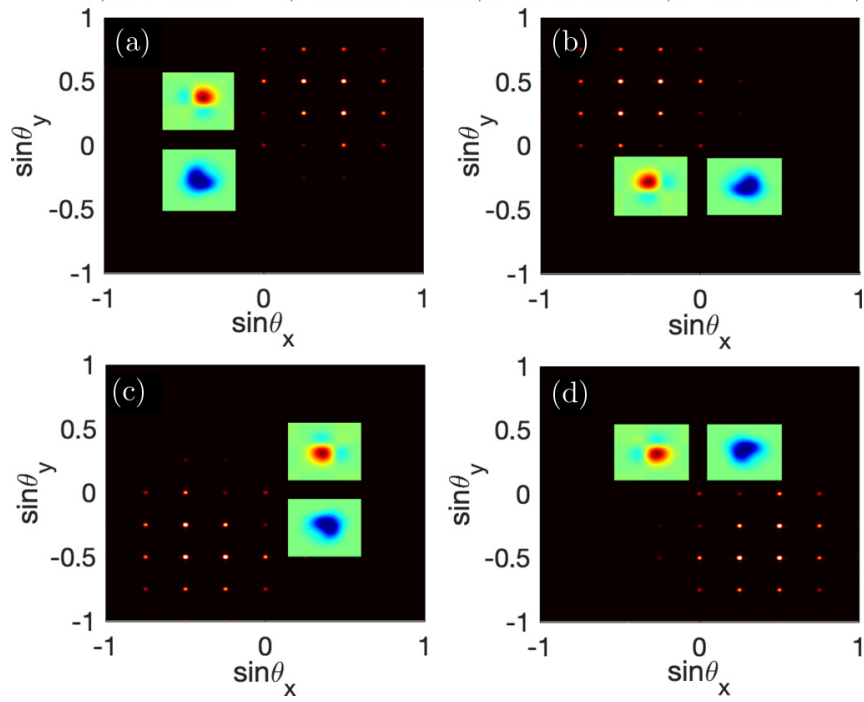

FIG. 7. Diffraction patterns of single diagonal symmetry attained for non-Hermitian modulations along both the $x$ and $y$ axes. Parameters are as in Fig. 3(b), except that (a) $\beta_{x}=\pi / 4$ and $\beta_{y}=\pi / 4$, (b) $\beta_{x}=-\pi / 4$ and $\beta_{y}=-3 \pi / 4$, (c) $\beta_{x}=-\pi / 4$ and $\beta_{y}=-\pi / 4$, and (d) $\beta_{y}=\pi / 4$ and $\beta_{y}=3 \pi / 4$. The insets show the corresponding absorption (upper or left) and dispersion (lower or right) distributions in a single square lattice.

to the $y(x)$ axis for $\beta_{y}=0$ and $\beta_{x}=\pi / 2$ [see Fig. 6(c)], and quadrants $I$ and $I I$ with respect to the $y(x)$ axis for $\beta_{y}=0$ and $\beta_{x}=-\pi / 2$ [see Fig. 6(d)]. We then control the single axial symmetric type of diffraction to realize $\pi / 2$ rotations through an appropriate choice of $\beta_{x}$ and $\beta_{y}$ among the values $\{0 ; \pm \pi / 2\}$. Such a control results in light diffraction symmetry along the direction displaying Hermitian modulation, yet asymmetry along the direction exhibiting $\mathcal{P} \mathcal{T}$-symmetry modulation.

Figure 7 shows that in case (ii), probe photons are diffracted into single quadrants, leading to single diagonal symmetry with respect to the $x=y$ or $x=-y$ line. Control of this diffraction pattern takes place through an appropriate choice of the shifts $\beta_{x}$ and $\beta_{y}$ among the values $\{ \pm \pi / 4, \pm 3 \pi / 4\}$, whereby the modulating factor $f(x, y)$ is neither odd nor even along both $x$ and $y$ axes. Once again, the hybrid non-Hermitian regime, realized via the modulations (i) and (ii) that are responsible, respectively, for the single axial symmetry in Fig. 6 and the single diagonal symmetry in Fig. 7 of far-field diffraction, hinges on the spatial interplay between the amplitude $\propto \chi_{p}^{I}(x, y)$ and phase $\propto \chi_{p}^{R}(x, y)$ modulations of the transmission $T_{L}(x, y)$; the probe photons are always diffracted into the quadrants where (1) $\chi_{p}^{I}(x, y)$ is positive (gain) when $\chi_{p}^{R}(x, y)$ is negative or (2) $\chi_{p}^{I}(x, y)$ is negative (loss) when $\chi_{p}^{R}(x, y)$ is positive, as shown by the insets.

The results shown in the last two sections are finally summed up in Table I, where the relationship between parameters and types of the 2D cross SW modulations (A) and (B), and patterns and symmetries of the corresponding far-field Fraunhofer diffraction are sketched. For completeness, we also give the results corresponding to the normal grating with 
TABLE I. Diffraction patterns and symmetries for four pure or hybrid cross modulations.

\begin{tabular}{llll}
\hline \hline SW pump phases & \multicolumn{1}{c}{ Modulation types } & Diffraction patterns & Diffraction symmetries \\
\hline$\beta_{x}=\pi / 2$ and $\beta_{y}=\pi / 2$ & Hermitian and Hermitian & 4 quadrants & Centrosymmetry \\
$\beta_{x}=0$ and $\beta_{y}=0$ & $\mathcal{P} \mathcal{T}$ symmetry and $\mathcal{P} \mathcal{T}$ symmetry & 2 diagonal quadrants & Double diagonal symmetry \\
$\beta_{x}=0$ and $\beta_{y}=\pi / 2$ & $\mathcal{P} \mathcal{T}$ symmetry and Hermitian & 2 adjacent quadrants & Single axial symmetry \\
$\beta_{x}=\pi / 4$ and $\beta_{y}=\pi / 4$ & Non-Hermitian and non-Hermitian & 1 quadrant & Single diagonal symmetry \\
\hline \hline
\end{tabular}

a Hermitian modulation along both the $x$ and $y$ axes $\left(\beta_{x}=\right.$ $\beta_{y}=\pi / 2$ ). In this case, photons can be scattered into all four quadrants with diffraction patterns of the centrosymmetry. It is not difficult to imagine that diffraction patterns exhibiting more involved symmetries or asymmetries could be observed upon choosing values for $\beta_{x}$ and $\beta_{y}$ different from those we have used. On more general grounds, our results are relevant to all-optical switching as well as optical imaging even for very weak probe fields. To this extent, as is often the case with driven atomic level configurations, it is worth noting that the 2D non-Hermitian cross gratings investigated here exhibit a certain versatility. In principle, they could be adapted to atom photonic crystal fiber interfaces, dealing even with few-photon light signals [61,62], or to solid interfaces with rare-earth-ion-doped crystals $[63,64]$ or NV color centers in diamond [65], where similar four-level $\mathcal{N}$ configurations exist.

\section{CONCLUSIONS}

The 2D optical lattices of driven cold atoms can provide an interesting venue to realize non-Hermitian EIG structures with various diffraction symmetry features. We have examined the far-field Fraunhofer diffraction off a thin 2D atomic lattice subject to transversely periodic cross modulations, including pure $\mathcal{P} \mathcal{T}$-symmetry ones as well as hybrid ones as a combination of Hermitian, $\mathcal{P} \mathcal{T}$-symmetry, and non-Hermitian modulations along two orthogonal axes. These spatial modulations, now realizable through standard laboratory routines [66], can be all-optically controlled to generate nontrivial far-field patterns that span from double diagonal symmetric diffraction in two diagonal quadrants to single axial symmetric diffraction in two adjacent quadrants and single diagonal symmetric diffraction in a single quadrant. These patterns appear to be fairy robust against standard sources of noises (fluctuations), e.g., in the atomic density or in the intensity of trapping or pumping lasers [67]. The origin of the peculiar diffraction patterns we anticipate is discussed in terms of the out-of-phase interplay of the amplitude $\propto \chi_{p}^{I}(x, y)$ and phase $\propto \chi_{p}^{R}(x, y)$ of the transmission $T(x, y)$ through the atomic lattice.

\section{ACKNOWLEDGMENTS}

This work is supported by the National Natural Science Foundation of China (Grants No. 10534002, No. 11674049, and No. 11704063), the Cooperative Program by the Italian Ministry of Foreign Affairs and International Cooperation (Grant No. PGR00960), and the National Natural Science Foundation of China (Grant No. 11861131001).
[1] C. M. Bender, Rep. Prog. Phys. 70, 947 (2007).

[2] R. El-Ganainy, K. G. Makris, D. N. Christodoulides, and Z. H. Musslimani, Opt. Lett. 32, 2632 (2007).

[3] C. Hang, G. Huang, and V. V. Konotop, Phys. Rev. Lett. 110, 083604 (2013).

[4] J. Sheng, M.-A. Miri, D. N. Christodoulides, and M. Xiao, Phys. Rev. A 88, 041803(R) (2013).

[5] L. Ge and H. E. Tureci, Phys. Rev. A 88, 053810 (2013).

[6] J.-H. Wu, M. Artoni, and G. C. La Rocca, Phys. Rev. Lett 113, 123004 (2014).

[7] J.-H. Wu, M. Artoni, and G. C. La Rocca, Phys. Rev. A 91, 033811 (2015).

[8] P. Peng, W. Cao, C. Shen, W. Qu, J. Wen, L. Jiang, and Y. Xiao, Nat. Phys. 12, 1139 (2016).

[9] F. Yang, Y.-C. Liu, and L. You, Phys. Rev. A 96, 053845 (2017).

[10] X. Wang and J.-H. Wu, Opt. Express 24, 4289 (2016).

[11] Y. L. Chuang, Ziauddin, and R. K. Lee, Opt. Express 26, 21969 (2018).

[12] V. V. Konotop and D. A. Zezyulin, Phys. Rev. Lett. 120, 123902 (2018).

[13] S. Longhi, Phys. Rev. Lett. 103, 123601 (2009).
[14] Y. Zhang, D. Zhang, Z. Zhang, C. Li, Y. Zhang, F. Li, M. R. Belić, and M. Xiao, Optica 4, 571 (2017).

[15] Y.-D. Chong, L. Ge, H. Cao, and A. D. Stone, Phys. Rev. Lett. 105, 053901 (2010).

[16] H. Hodaei, M.-A. Miri, M. Heinrich, D. N. Christodoulides, and M. Khajavikhan, Science 346, 975 (2014).

[17] L. Feng, Z.-J. Wong, R.-M. Ma, Y. Wang, and X. Zhang, Science 346, 972 (2014).

[18] B. Peng, Ş. K. Özdemir, S. Rotter, H. Yilmaz, M. Liertzer, F. Monifi, C. M. Bender, F. Nori, and L. Yang, Science 346, 328 (2014).

[19] H. Jing, Ş. K. Özdemir, X.-Y. Lü, J. Zhang, L. Yang, and F. Nori, Phys. Rev. Lett. 113, 053604 (2014).

[20] M. Kulishov, J. M. Laniel, Ni. Bélanger, J. Azaña, and D. V. Plant, Opt. Express 13, 3068 (2005).

[21] Z. Lin, H. Ramezani, T. Eichelkraut, T. Kottos, H. Cao, and D. N. Christodoulides, Phys. Rev. Lett. 106, 213901 (2011).

[22] X. Yin and X. Zhang, Nat. Mater. 12, 175 (2013).

[23] L. Feng, Y.-L. Xu, W. S. Fegadolli, M.-H. Lu, J. E. B. Oliveira, V. R. Almeida, Y.-F. Chen, and A. Scherer, Nat. Mater. 12, 108 (2013). 
[24] M. Kulishov and B. Kress, Opt. Express 20, 29319 (2012).

[25] M. Kulishov, B. Kress, and H. F. Jones, Opt. Express 22, 23164 (2014).

[26] M. Kulishov, H. F. Jones, and B. Kress, Opt. Express 23, 9347 (2015).

[27] S. Longhi, Phys. Rev. A 81, 022102 (2010).

[28] M. Fleischhauer, A. Imamoglu, and J. P. Marangos, Rev. Mod. Phys. 77, 633 (2005).

[29] A. André and M. D. Lukin, Phys. Rev. Lett. 89, 143602 (2002).

[30] M. Artoni and G. C. La Rocca, Phys. Rev. Lett. 96, 073905 (2006).

[31] Y. Zhang, Y.-M. Liu, X.-D. Tian, T.-Y. Zheng, and J.-H. Wu, Phys. Rev. A 91, 013826 (2015).

[32] M. Bajcsy, A. S. Zibrov, and M. D. Lukin, Nature (London) 426, 638 (2003).

[33] J.-H. Wu, M. Artoni, and G. C. La Rocca, Phys. Rev. A 82, 013807 (2010).

[34] J. L. Everett, G. T. Campbell, Y.-W. Cho, P. Vernaz-Gris, D. Higginbottom, O. Pinel, N. P. Robins, P. K. Lam, and B. C. Buchler, Nat. Phys. 13, 68 (2017).

[35] H.-Y. Ling, Y.-Q. Li, and M. Xiao, Phys. Rev. A 57, 1338 (1998).

[36] M. Mitsunaga and N. Imoto, Phys. Rev. A 59, 4773 (1999).

[37] G. C. Cardoso and J. W. R. Tab osa, Phys. Rev. A 65, 033803 (2002).

[38] L. Zhao, W. Duan, and S. F. Yelin, Phys. Rev. A 82, 013809 (2010).

[39] M. V. Berry, J. Phys. A 31, 3493 (1998).

[40] V. V. Konotop and B. I. Mantsyzov, Opt. Express 24, 26146 (2016).

[41] Y.-M. Liu, X.-D. Tian, X. Wang, D. Yan, and J.-H. Wu, Opt. Lett. 41, 408 (2016).

[42] S. Asghar, Ziauddin, S. Qamar, and S. Qamar, Phys. Rev. A 94, 033823 (2016).

[43] Y.-M. Liu, F. Gao, C.-H. Fan, and J.-H. Wu, Opt. Lett. 42, 4283 (2017).

[44] T. Shui, W.-X. Yang, S. Liu, L. Li, and Z. Zhu, Phys. Rev. A 97, 033819 (2018).

[45] S.-C. Tian, R.-G. Wan, L.-J. Wang, S.-L. Shu, H.-Y. Lu, X. Zhang, C.-Z. Tong, J.-L. Feng, M. Xiao, and L.-J. Wang, Opt. Express 26, 32918 (2018).

[46] F. Gao, Y.-M. Liu, X.-D. Tian, C.-L. Cui, and J.-H. Wu, Opt. Express 26, 33818 (2018).

[47] D.-D. Ma, D.-M. Yu, X.-D. Zhao, and J. Qian, Phys. Rev. A 99, 033826 (2019).

[48] N.-F. Yu and F. Capasso, Nat. Mater. 13, 139 (2014).

[49] C. Pfeiffer and A. Grbic, Phys. Rev. Appl. 2, 044012 (2014).
[50] A. M. Shaltout, V. M. Shalaev, and M. L. Brongersma, Science 364, eaat3100 (2019).

[51] I. Bloch, Nat. Phys. 1, 23 (2005).

[52] The consideration of other low-lying states would not change our results much, yet would make the treatment overly complicated. We have checked that our conclusions hold even for a Lorentzian distribution of atomic density.

[53] Here, $N_{0}$ is basically determined as atoms are preliminarily loaded into a magneto-optical trap. But it is viable to get a higher (lower) $N_{0}$ and a smaller (larger) $\sigma_{x, y}$ by ramping up (down) the depth of the optical lattice traps.

[54] C. Gardiner and P. Zoller, Quantum Noise: A Handbook of Markovian and Non-Markovian Quantum Stochastic Methods with Applications to Quantum Optics, 2nd ed., Springer Series in Synergetics (Springer, New York, 2004).

[55] For typical values of trap beam waist $\sim 80 \mu \mathrm{m}$ and probe beam wavelength $\sim 1 \mu \mathrm{m}$, the Fraunhofer diffraction is valid at observation distances larger than Rayleigh length $\sim 20 \mathrm{~mm}$. In this case, our 2D atomic lattice of thickness $L=100 \mu \mathrm{m}$ behaves like a rather thin grating in which propagation effects could be deemed less relevant.

[56] L. Wang, F.-X. Zhou, P.-D. Hu, Y.-P. Niu, and S.-Q. Gong, J. Phys. B: At. Mol. Opt. Phys. 47, 225501 (2014).

[57] J.-C. Wu and B.-Q. Ai, J. Phys. B: At. Mol. Opt. Phys. 48, 115504 (2015).

[58] For the specific driving conditions that we examine, the susceptibility in Eq. (2) is sufficiently small so that the associated refractive index $n=\sqrt{1+\chi_{p}}$ is very close to its vacuum value of 1.0 , and hence boundary conditions can be safely neglected in calculating the transmission [59].

[59] M. Artoni, G. C. La Rocca, F. Cataliotti, and F. Bassani, Phys. Rev. A 63, 023805 (2001).

[60] If one changes, e.g., $\beta_{x} \rightarrow \beta_{x}+\pi$ or $\beta_{y} \rightarrow \beta_{y}+\pi$ [see Eq. (6)], then $f(x, y) \rightarrow-f(x, y)$.

[61] V. Venkataraman, K. Saha, P. Londero, and A. L. Gaeta, Phys. Rev. Lett. 107, 193902 (2011).

[62] M. Artoni and R. Loudon, Phys. Rev. A 57, 622 (1998).

[63] Y.-F. Fan, H.-H. Wang, R. Wang, X.-G. Wei, A.-J. Li, Z.-H. Kang, J.-H. Wu, H.-Z. Zhang, H.-L. Xu, and J.-Y. Gao, New J. Phys. 13, 123008 (2011).

[64] Q.-Y. He, Y. Xue, M. Artoni, G. C. La Rocca, J.-H. Xu, and J.-Y. Gao, Phys. Rev. B 73, 195124 (2006).

[65] M. W. Doherty, N. B. Manson, P. Delaney, F. Jelezko, J. Wrachtrup, and L. C. L. Hollenberg, Phys. Rep. 528, 1 (2013).

[66] Z. Y. Zhang, L. Yang, J. L. Feng, J. T. Sheng, Y. Q. Zhang, Y. P. Zhang, and M. Xiao, Laser. Photon. Rev. 12, 1800155 (2018).

[67] We have checked that appreciable influences on the 2D diffraction patterns can only be observed for $\sim 5 \%(\sim 10 \%)$ or larger random fluctuations on $N_{0}\left(\delta \Omega_{c}\right)$. 